\title{
Impact of cigarette smoke and aerobic physical training on histological and molecular markers of prostate health in rats
}

\author{
A.S.C. Veras $\mathbb{1}^{1}{ }^{1}$, D.B. Baptista ${ }^{1}$, N.J. dos Santos $\mathbb{1}^{3}{ }^{3}$, H.H.A. Thorpe ${ }^{4}$, P.M. Seraphim $\mathbb{1}^{5}$, \\ A.R. Florido Neto $^{6}$, and G.R. Teixeira (i) $^{1,2}$ \\ ${ }^{1}$ Programa de Pós-Graduação em Ciências da Motricidade, Universidade Estadual Paulista, Presidente Prudente, SP, Brasil \\ ${ }^{2}$ Departamento de Educação Física, Universidade Estadual Paulista, Faculdade de Ciências e Tecnologia, UNESP, \\ Presidente Prudente, SP, Brasil \\ ${ }^{3}$ Programa de Pós-Graduação em Biologia Celular e Estrutural, Universidade de Campinas, Campinas, SP, Brasil \\ ${ }^{4}$ Department of Biomedical Sciences, Ontario Veterinary College, University of Guelph, Guelph, ON, Canada \\ ${ }^{5}$ Departamento de Fisioterapia, Faculdade de Ciências e Tecnologias, Universidade Estadual Paulista, \\ Presidente Prudente, SP, Brasi \\ ${ }^{6}$ Programa de Pós-Graduação em Fisiologia, Universidade de São Paulo, São Paulo, SP, Brasil
}

\begin{abstract}
Recent evidence suggests that aerobic physical training may attenuate the deleterious effects of cancer risk factors, including smoking. We investigated the effects of cigarette smoke inhalation and aerobic physical training on the expression of steroid receptors and inflammatory and apoptotic proteins in the prostate. Forty male Wistar rats were distributed in four groups: control $(C O)$, exercise (EXE), cigarette smoke exposure (CS), and cigarette smoke exposure with exercise (CS + EXE). For eight weeks, animals were repeatedly exposed to cigarette smoke for $30 \mathrm{~min}$ or performed aerobic physical training either with or without the cigarette smoke inhalation protocol. Following these experiments, we analyzed prostate epithelial morphology and prostatic expression of androgen (AR) and glucocorticoid receptors (GR), insulin-like growth factor (IGF-1), B-cell lymphoma-2 (BCL-2), BCL-2-associated X protein (BAX), interleukin-6 (IL-6), tumor necrosis factor-alpha (TNF- $\alpha$ ), and nuclear factor-kappa $\mathrm{B}(\mathrm{NF}-\kappa \mathrm{B})$ via immunohistochemistry. Cigarette smoke exposure stimulated the expression of AR, IGF-1, BCL-2, and NF- $\kappa B$ while downregulating $\mathrm{BAX}, \mathrm{IL}-6$, and TNF- $\alpha$ labeling in the prostate. In contrast, aerobic physical training attenuated cigarette smoke-induced changes in AR, GR, IGF-1, BCL-2, IL-6, TNF- $\alpha$, and NF- $\kappa$ B. This suggests that cigarette smoke stimulates inflammation and reduces apoptosis, culminating in increased prostatic epithelial and extracellular matrices, whereas physical training promoted beneficial effects towards maintaining normal prostate morphology and protein levels.
\end{abstract}

Key words: Inflammation; Androgen receptor; Growth factors; Endurance exercise; BAX; BCL-2

\section{Introduction}

Cigarette smoking is considered one of the greatest risk factors in the development of cardiovascular disease and is a potent metabolic stimulator in the progression of various types of cancer, including prostate cancer (1). Studies indicate that secondhand or sidestream smoke contains higher concentrations of toxic components than mainstream smoke, and secondhand smoke exposure is a factor in the development of smoking-related diseases (2). The toxic components of cigarette smoke alter the mitogenic and anti-apoptotic properties of hormonal peptides, resulting in changes in metabolism through dysregulation of proliferation, epithelial differentiation, and apoptotic inhibition (3).
Current data provide convincing evidence that physical exercise reduces the risk of lung, prostate, and endometrial diseases (4). Although the most optimal intensity, volume, and modality of exercise to combat disease have yet to be established in the literature, there are reports that moderate physical exercise increases apoptosis in cancer cells (5). Tobacco-induced alterations to prostate tissue proliferation and chronic inflammation have been reported in clinical populations (6). While it is possible that exercise promotes beneficial reductions in prostate cell proliferation and inflammation caused by smoke exposure, there are currently no preclinical studies evaluating the interaction between exercise, prostatic health, and smoking. Notably,

Correspondence: G.R. Teixeira: <giovana.rampazzo@unesp.br> 
physical exercise can modulate cellular activity in the rat prostate by reducing androgen receptor (AR) activation, which in turn reduces cellular proliferation (5). Glucocorticoid receptor (GR) activation is also implicated in prostatic health and exercise, as glucocorticoid administration in rodents conduces hormonal regulation in the prostate and aerobic physical training increases plasma glucocorticoid levels, consequently inducing intracellular GR activation (7). Glucocorticoids such as cortisol in humans and corticosterone in rats are steroid hormones produced by the adrenal glands and regulated by the hypothalamicpituitary-adrenal axis. These molecules play a fundamental role in controlling physiological processes, including development, inflammation, and stress responses (8). Evidence suggests that glucocorticoids are integral to programmed cell death and the inhibition of cell proliferation in androgenindependent prostatic adenocarcinomas (7).

Although cigarette smoking is a clear factor in the development of prostate disorders, the molecular mechanisms of smoking-related carcinogenesis are complex and involve the dysregulation of many proteins and pathways. Kenfield and colleagues (9) evaluated correlations between inflammatory features and smoking in 5,366 prostate cancer patients and found that smoking and inflammation substantially increased mortality rates and cancer recurrence. Substantial increases in levels of the transcription factor complex nuclear factor kappa-light-chain-enhancer of activated $B$ cells (NF- $\mathrm{B}$ ) by pro-inflammatory cytokines, including interleukin-1 (IL-1), IL-6, and tumor necrosis factor $\alpha(\mathrm{TNF}-\alpha)$, have been observed in plasma and prostate samples of tobacco users (6). Reactive oxygen species generated by cigarette smoking have been linked to an increase in inflammatory gene expression through the induction of NF- $\kappa \mathrm{B}$ and proinflammatory mediators, leading to inflammation and epithelial disease progression (8). Aerobic physical training of moderate-intensity (40-60\% $\mathrm{VO}_{2}$ max) decreases circulating levels of inflammatory cytokines IL- 6 and TNF- $\alpha$ while concurrently increasing plasma levels of IL-10 (10). However, while cigarette smoking and aerobic physical exercise may independently alter prostate cell metabolism, the relationship between smoking and physical exercise on prostatic health is unclear. We investigated the ability of cigarette smoke inhalation and aerobic physical training, alone or in combination, to modulate inflammatory, apoptotic, and steroid receptor protein expression in the rat prostate. We found that exercise had beneficial prospects in maintaining prostate health, even in animals chronically exposed to cigarette smoke.

\section{Material and Methods}

\section{Animals and experimental design}

All animal protocols were approved by the Ethics Committee on the Use of Animals (CEUA) of the School of Technology and Sciences at São Paulo State University (UNESP), campus of Presidente Prudente (Protocol No. 1/2012). All procedures were conducted following the guidelines for experimentation with animals according to the Guide to the Care and Use of Laboratory Animals published by the United States National Institutes of Health.

Forty post-pubertal Wistar rats from the Central Animal Laboratory at UNESP Botucatu were maintained at an average temperature of $22 \pm 2^{\circ} \mathrm{C}$ under a 12-h light/dark cycle in solid-bottomed polyethylene cages $(40 \times 30 \times$ $15 \mathrm{~cm}$ ). Animals had ad libitum access to filtered tap water and standard food chow. Prior to testing, animals were randomly divided into one of four groups: control (CO; $n=10$ ), which remained inactive for the duration of the study; cigarette smoke inhalation $(C S ; n=8$, as two animals died during the course of the study), which were subjected to cigarette smoke inhalation; exercise (EXE; $n=10$ ), which were subjected to an aerobic physical exercise protocol as described below; and cigarette smoke inhalation with exercise (CS + EXE; $n=10$ ), which were exposed to both smoke inhalation and the aerobic physical exercise protocol (Figure 1).

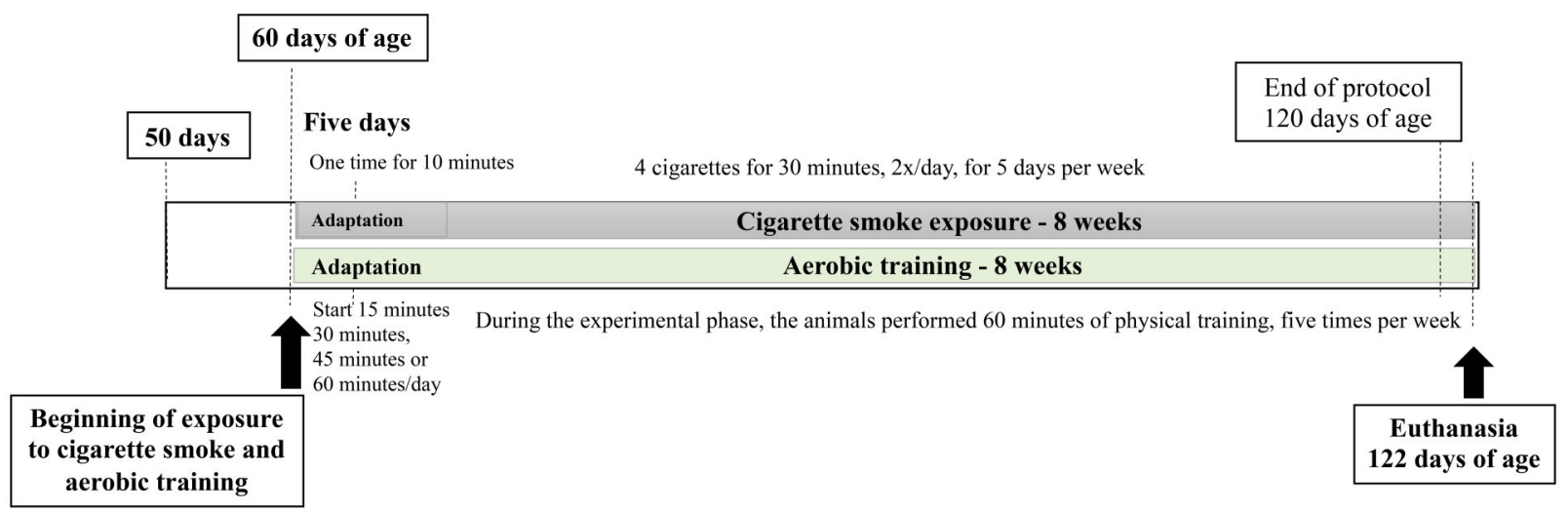

Figure 1. Timeline of experimental design during the aerobic training and cigarette smoke exposure. 


\section{Exposure to cigarette smoke}

At 60 days of age, animals in the CS and CS + EXE groups were exposed to cigarette smoke in two phases: adaptation and experimental. In the adaptation phase (first five days of experimentation), the animals were exposed to the smoke of two cigarettes for 10 min once per day. During the experimental phase, animals were exposed to smoke from four cigarettes for 30 min twice per day, five days per week for eight weeks. At these doses, animals were exposed to $250-350 \mathrm{ppm}$ of carbon monoxide (11). The animals were placed in one of two compartments in a two-sided chamber, and the cigarettes were burned separately in the other compartment. An air compressor (10 L/min) was used to ensure the passage of smoke to the animal through a hole connecting the two compartments. Commercially-available cigarettes (Malboro Red ${ }^{\mathbb{R}}$ ) were composed of a mixture of tobacco, sugars, cigarette paper, and vegetable extracts; flavoring agents were used. As reported on the packaging, each cigarette produced $10 \mathrm{mg}$ of tar, $0.8 \mathrm{mg}$ of nicotine, and $10 \mathrm{mg}$ of carbon monoxide when burned.

\section{Physical training protocol}

At 60 days of age, the exercise groups performed aerobic physical training on a treadmill at $10 \mathrm{~m} / \mathrm{min}$ as previously described (12). Animals underwent an adaptation phase for one week followed by an eight-week experimental phase. During the adaptation phase, the animals performed aerobic physical training on a treadmill for either $15,30,45$, or 60 min per day. During the experimental phase, the animals performed 60 min of physical training five times per week.

\section{Body mass index and nutritional analyses}

Body weights were measured using a Shimadzu electronic scale (model BL3200H, Japan) once per week throughout the study. Energy intake (El; $3 \mathrm{kcal} / \mathrm{g})$, which was assessed weekly, was used to calculate ingested energy $(\mathrm{El}$ (kcal/100 g per day) = average food intake [g] per day $\times 100 /$ animal weight $\times 3 \mathrm{kcal}[\mathrm{g} / 100 \mathrm{~g}$ per day]). Feed efficiency (FE) was calculated as $\mathrm{FE}(\mathrm{g} / \mathrm{kcal})=$ mean weight gain / El, as previously described (13). To evaluate weight gain, we calculated body mass gain ( $\Delta=$ final weight starting weight). Body mass index (BMI) was evaluated using the weight and nose-to-anus length (BMI: body weight $[\mathrm{g}] /$ length $\left.\left[\mathrm{cm}^{2}\right]\right)(13)$.

\section{Biochemical analyses}

Blood samples were drawn from the portal vein after $12 \mathrm{~h}$ of fasting. The collection was done using a syringe without an anticoagulant. After collection, blood was centrifuged for $10 \mathrm{~min}$ at $1008 \mathrm{~g}$ and stored at $-80^{\circ} \mathrm{C}$. Triglyceride content, total cholesterol, and glucose serum levels were determined using colorimetry assay (Labtest, Brazil; ZenBio, Inc., USA).

\section{Histopathological analysis}

Prostate tissue was fixed in formalin solution for $24 \mathrm{~h}$, underwent a routine of paraplastic inclusion, and cut into $5-\mu \mathrm{m}$ sections. The sections were stained with hematoxylin and eosin (HE) to analyze histological morphology in the prostate. Using a Weibel reticle of 160 points, the stereology (acini, epithelium, and stroma) of prostate cross-sections was quantified across 10 images per slide, and 10 slides were analyzed per prostate collected. Images were obtained using an AxioCam ER.c 5s (Zeiss Primo Star, Germany) photomicroscope with a $40 \times$ objective at the Department of Physical Education of São Paulo State University (UNESP).

\section{Immunohistochemistry protocol}

Prostate sections were mounted on glass slides, dewaxed with xylene and alcoholic gradations, and washed in running water. The slides were placed in a microwave oven in sodium citrate buffer $(0.01 \mathrm{M}, \mathrm{pH} 6.0)$ for $15 \mathrm{~min}$. Thereafter, slides were submerged in a $3 \%$ hydrogen peroxide solution diluted in methanol for $15 \mathrm{~min}$ and a $3 \%$ bovine serum albumin solution for one hour for protein blockade. Sections were then incubated with AR (N-20, sc-816, Santa Cruz Biotechnology, USA, 1:100), GR (M-20, sc-1004, Santa Cruz Biotechnology, 1:100), BCL-2 (N-19, sc-492, Santa Cruz Biotechnology, 1:250), BAX (P19, sc-526, Santa Cruz Biotechnology, 1:300), IL-6 (M-19, sc-1265, Santa Cruz Biotechnology, 1:60), TNF- $\alpha$ (N-19, sc-1350, Santa Cruz Biotechnology, 1:80), and NF-кB (A, sc-109, p65 subunit, Santa Cruz Biotechnology, 1:100) primary antibodies in a wet chamber overnight at $4^{\circ} \mathrm{C}$. After incubation, sections were washed in sodium phosphate buffer and incubated for two hours with a secondary antibody (mouse anti-goat immunoglobulin G-horseradish peroxidase IgG-HRP, sc-2354, Santa Cruz Biotechnology, 1:200; mouse anti-rabbit IgG-HRP, sc-2357, Santa Cruz Biotechnology, 1:200). The sections were then exposed to diaminobenzidine (DAB) and counterstained with hematoxylin. For all markers, positive and negative controls were performed (14). As the positive control, we used several dilutions of the antibody in the prostate tissue to adjust the protocol associated with the negative control.

The intensity of immunoreactivity to IGF-1, BCL-2, BAX, IL-6, TNF- $\alpha$, and NF- $\kappa B$ antigens was examined in 10 fields per prostate using ImageJ software (version $1.50 \mathrm{i}, \mathrm{NIH}$, USA) by analyzing the percentage of tissue labeling in each field and the absorbance of IGF-1, BCL-2, BAX, IL-6, TNF- $\alpha$, and NF- $\kappa B$ immunopositive cells were used for percentage for area.

For the AR and GR quantification, the labeling indexes for each group were estimated as the percentage of stained-positive epithelial cells. The average AR and GR indexes in each group were then obtained in 1,000 secretory epithelial cells per animal from 10 sections of the intermediate region of the ventral prostate. 


\section{Data analyses}

The Shapiro-Wilk test was used to verify the normality and homogeneity of the data. Statistical analyses of tissue protein expression and anthropometric parameters were performed with one-way ANOVA and complemented with Tukey's multiple comparison test between group means. Statistical analysis of BMI values was conducted using a one-way ANOVA and Fisher's exact test. Correlation coefficients were calculated using Pearson's correlation coefficient test. Results are reported as means $\pm S D$, and $\mathrm{P}<0.05$ was considered statistically significant. All statistical analysis was performed using SPSS software v.25 (IBM, USA).

\section{Results}

\section{Body mass index, nutritional, and biochemical parameters}

In this study, we demonstrated that exposure to cigarette smoke in both the CS and CS + EXE conditions reduced weight gain compared to the $\mathrm{CO}$ group and that the CS group alone showed significant reductions in total ingested energy relative to all other treatment groups. However, feed efficiency was also reduced in the CS + EXE group compared with EXE despite no significant difference in final animal weight across groups. Calculations of BMI showed that EXE and CS conditions resulted in lower values than the CO group, although interestingly, no reduction in BMI was observed in the CS+EXE condition. The EXE group had higher triglyceride plasma concentrations than the CS and CO groups, and the CS and CS + EXE groups had higher total cholesterol levels than the CO and EXE groups (Table 1).

\section{Histological changes}

To determine if cigarette smoke exposure and exercise, alone or in combination, altered the histological profile of the prostate, we examined prostatic epithelial volume and stroma as well as related stereology and proliferative protein expression. Secretory epithelium in the prostate with columnar and basal cells and other cells in the glandular stroma had a surface area of $26.6 \%$ epithelium, $57.6 \%$ acinar lumen, and $15.6 \%$ stroma in the $\mathrm{CO}$ group (Figure 2A, B, I). In the EXE group, the epithelium surface area was reduced to $24.3 \%$, and the proportion of acinar lumen increased to $63.9 \%$, though these changes were not significantly different from the $\mathrm{CO}$ condition (Figure 2C, D, I). Prostate columnar epithelium surface area in the CS group presented intraepithelial neoplasia as the proportion of epithelium was increased to $42 \%$, significantly greater than all other conditions (Figure 2E, F, I, $\mathrm{P}=0.03$ ). Interestingly, the exercise paradigm appeared to mitigate the increased prostatic epithelium caused by cigarette smoke exposure. In addition to epithelium changes, lumen decreases were also observed following smoke exposure in the CS (35.5\%) and CS + EXE $(32.7 \%)$ conditions $(P=0.04)$. Although no significant lumen differences were observed between the CS and CS + EXE groups, the higher lumen content in the CS + EXE condition that was more reminiscent of the CO group may suggest a protective effect of exercise against smokeinduced prostate changes. However, despite no significant differences in stroma content across all conditions, both the CS and CS + EXE groups demonstrated a higher average stromal surface area than the $\mathrm{CO}$ and EXE groups (Figure 2l).

\section{Effects of cigarette smoke exposure and aerobic} exercise on steroid receptors and apoptotic proteins

In the CS group, AR and IGF-1 protein expression were significantly higher than in the other groups, whereas AR expression was lowest in the EXE group; IGF-1 expression was lowest in the CS + EXE and EXE conditions (Figure $3 A$ to $D, M ; I-L, O$ ). Representative images

Table 1. Anthropometry and nutritional and biochemical parameters of control animals (CO), animals submitted to exercise (EXE), cigarette smoke exposure (CS), and cigarette smoke exposure with exercise (CS +EXE).

\begin{tabular}{lccccc}
\hline & CO & EXE & CS & CS +EXE & P-value \\
\hline Final weight (g) & $459.40 \pm 44.21$ & $435.80 \pm 35.21$ & $434.00 \pm 63.50$ & $437.10 \pm 34.12$ & 0.553 \\
Food consumption (g/day) & $27.95 \pm 1.40^{\mathrm{c}}$ & $27.80 \pm 1.6$ & $26.03 \pm 1.76$ & $28.49 \pm 1.43^{\mathrm{c}}$ & 0.008 \\
Ingested energy (kcal/day) & $81.07 \pm 4.07^{\mathrm{c}}$ & $80.64 \pm 4.77^{\mathrm{c}}$ & $75.48 \pm 5.11$ & $82.63 \pm 4.14^{\mathrm{c}}$ & 0.008 \\
Feed efficiency (\%) & $77.48 \pm 28.70$ & $86.18 \pm 20.70$ & $71.68 \pm 28.22$ & $52.43 \pm 19.90^{\mathrm{b}}$ & 0.030 \\
Weight gain (g) & $98.30 \pm 23.58$ & $92.30 \pm 27.15$ & $78.42 \pm 11.19^{\mathrm{a}}$ & $69.30 \pm 27.47^{\mathrm{a}}$ & 0.049 \\
Body mass index (g/cm ${ }^{2}$ ) & $0.73 \pm 0.04$ & $0.68 \pm 0.02^{\mathrm{a}}$ & $0.68 \pm 0.04^{\mathrm{a}}$ & $0.72 \pm 0.05$ & 0.048 \\
Weight of prostate (g) & $0.670 \pm 0.08$ & $0.647 \pm 0.07$ & $0.581 \pm 0.09$ & $0.726 \pm 0.09$ & 0.140 \\
Relative weight of prostate & $0.158 \pm 0.03$ & $0.157 \pm 0.01$ & $0.137 \pm 0.01$ & $0.174 \pm 0.01$ & 0.190 \\
Triglycerides (mg/dL) & $82.46 \pm 9.46^{\mathrm{b}}$ & $111.85 \pm 23.92$ & $83.41 \pm 9.92^{\mathrm{b}}$ & $94.20 \pm 11.14$ & 0.009 \\
Cholesterol (mg/dL) & $68.24 \pm 5.23^{\mathrm{c.d}}$ & $72.84 \pm 5.61^{\mathrm{c}}$ & $87.95 \pm 13.31$ & $83.15 \pm 17.22$ & 0.028 \\
Glucose $(\mathrm{mg} / \mathrm{dL})$ & $111.52 \pm 9.75$ & $91.33 \pm 14.60$ & $103.07 \pm 18.15$ & $100.76 \pm 17.81$ & 0.193 \\
\hline
\end{tabular}

Data are reported as means $\pm \mathrm{SD}$. ${ }^{\mathrm{a}} \mathrm{P}<0.05$ vs $\mathrm{CO}$; ${ }^{\mathrm{b}} \mathrm{P}<0.05$ vs EXE; ${ }^{\mathrm{C}} \mathrm{P}<0.05$ vs $\mathrm{CS}$; ${ }^{\mathrm{d}} \mathrm{P}<0.05$ vs CS + EXE, one-way ANOVA with Tukey's multiple comparisons post hoc test. 

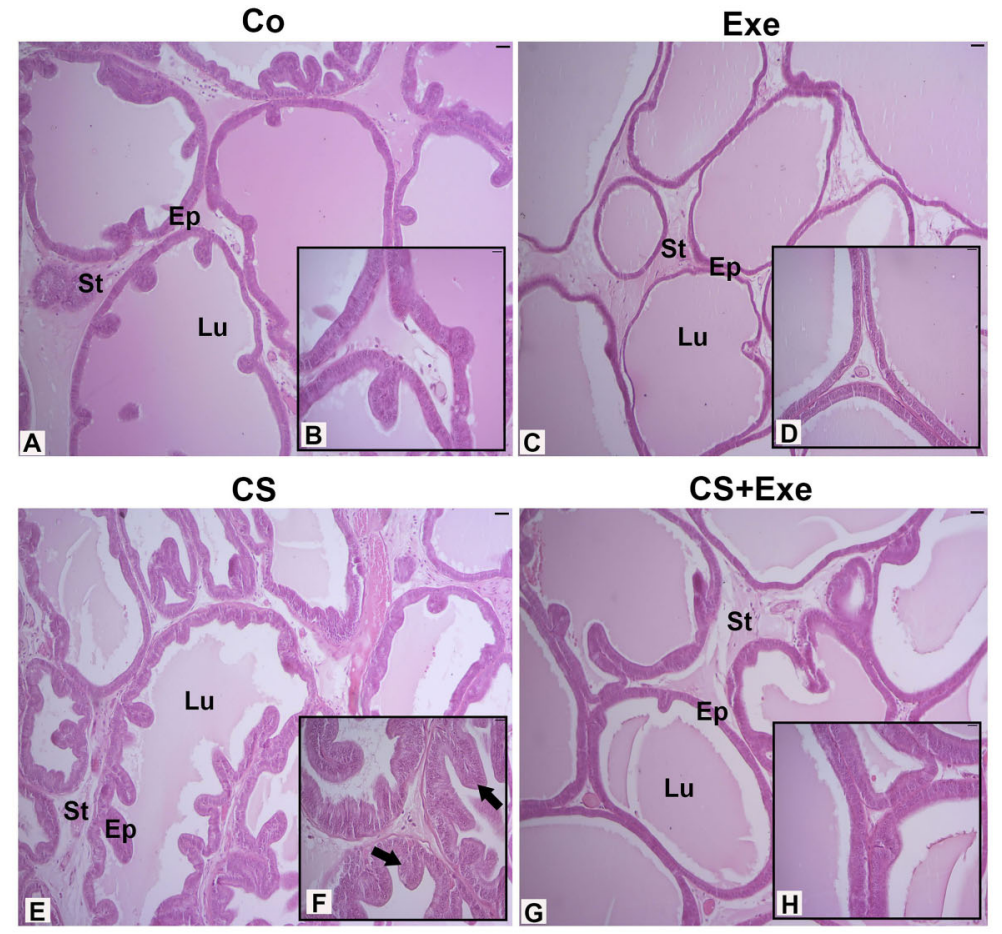

CS+Exe

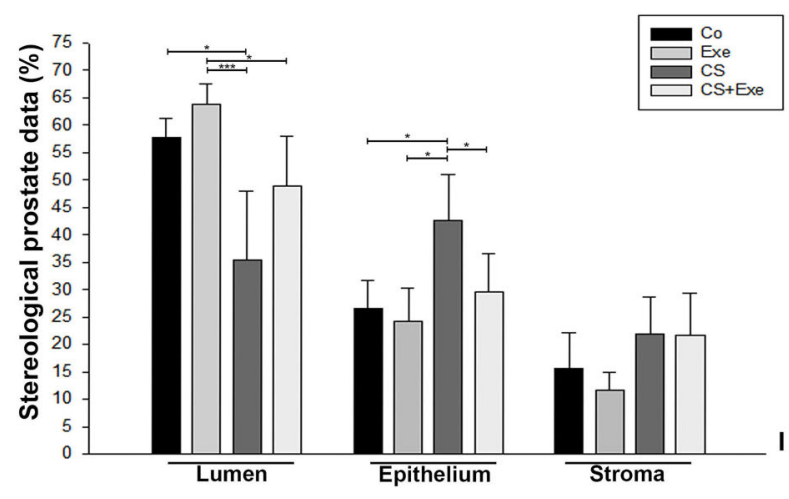

Figure 2. Histological analysis and quantification of stereology in the ventral prostate as a percentage of total prostate surface area from Wistar rats in the control group (Co) $(n=10, A$ and B), exercise only group (Exe) $(n=10, C$ and $\mathbf{D})$, smoke exposure only group (CS) ( $n=8, E$ and $\mathbf{F})$, and exercise with smoke exposure group (CS + Exe) $(n=10, \mathbf{G}$ and $\mathbf{H})$. Images were captured at $10 \times$ magnification (scale bars, $50 \mu \mathrm{m}$ ) and enlarged to $40 \times$ magnification (scale bars, $20 \mu \mathrm{m}$ ) in the insets. Arrows indicate locations with prostatic intraepithelial neoplasia. Data are reported as mean percentage of surface area \pm SD. The Weibel reticle of 160 points was used for morphometric analysis and the stereology (acini, epithelium, and stroma) of prostate cross-sections were quantified across 10 images per slide, with 10 slides analyzed per prostate collected. ${ }^{*} \mathrm{P}<0.05$, ${ }^{* * *} \mathrm{P}<0.001$, one-way ANOVA supplemented with Tukey multiple comparisons post hoc test. St: Stroma; Ep: Epithelium; Lu: Lumen. of GR expression in the prostate of the CS group exemplify the low GR content in prostatic epithelium relative to all other conditions (Figure $3 \mathrm{E}$ to $\mathrm{H}, \mathrm{N}$ ). In contrast, GR expression in the EXE and CS + EXE conditions were comparable to observations in the $\mathrm{CO}$ condition, further suggesting a protective effect of exercise in prostate receptor expression. Similar to observations of $A R$ and IGF-1 expression, levels of the anti-apoptotic protein BCL2 and the ratio of $B C L-2 / B A X$, a marker of prostate tumor proliferative potential, were higher in animals submitted to the CS protocol than in all other groups (Figure 4A to D, I, $\mathrm{K})$. In contrast, BAX expression was highest in the EXE group relative to all other conditions (Figure 4E to $H, J$ ). This suggested that smoke exposure increased antiapoptotic protein expression, dysregulated the ratio of BCL-2/BAX, and increased AR and IGF-1, inducers of cell proliferation. Exercise mitigated these changes.
Furthermore, a positive correlation between epithelium volume and BCL-2 expression was observed under cigarette exposure conditions ( $r=0.447, \mathrm{P}=0.048)$.

\section{Physical exercise reduced prostatic proinflammatory cytokines}

Representative images of prostatic IL-6, TNF- $\alpha$, and $N F-\kappa B$ expression in each group are presented in Figure $5 A$ to $L$. The EXE and CS + EXE protocols reduced the expression of pro-inflammatory IL-6 compared with expression in the $\mathrm{CO}$ and CS groups (Figure $5 \mathrm{M}$ ). Additionally, the EXE and CS + EXE groups also showed reduced TNF- $\alpha$ expression compared with the CS group (Figure $5 \mathrm{~N}$ ). In contrast, the CS group presented the highest NF-kB expression compared with all other groups (Figure 5O). These results suggested smoke exposure promoted inflammation and decreased GR expression. However, aerobic 

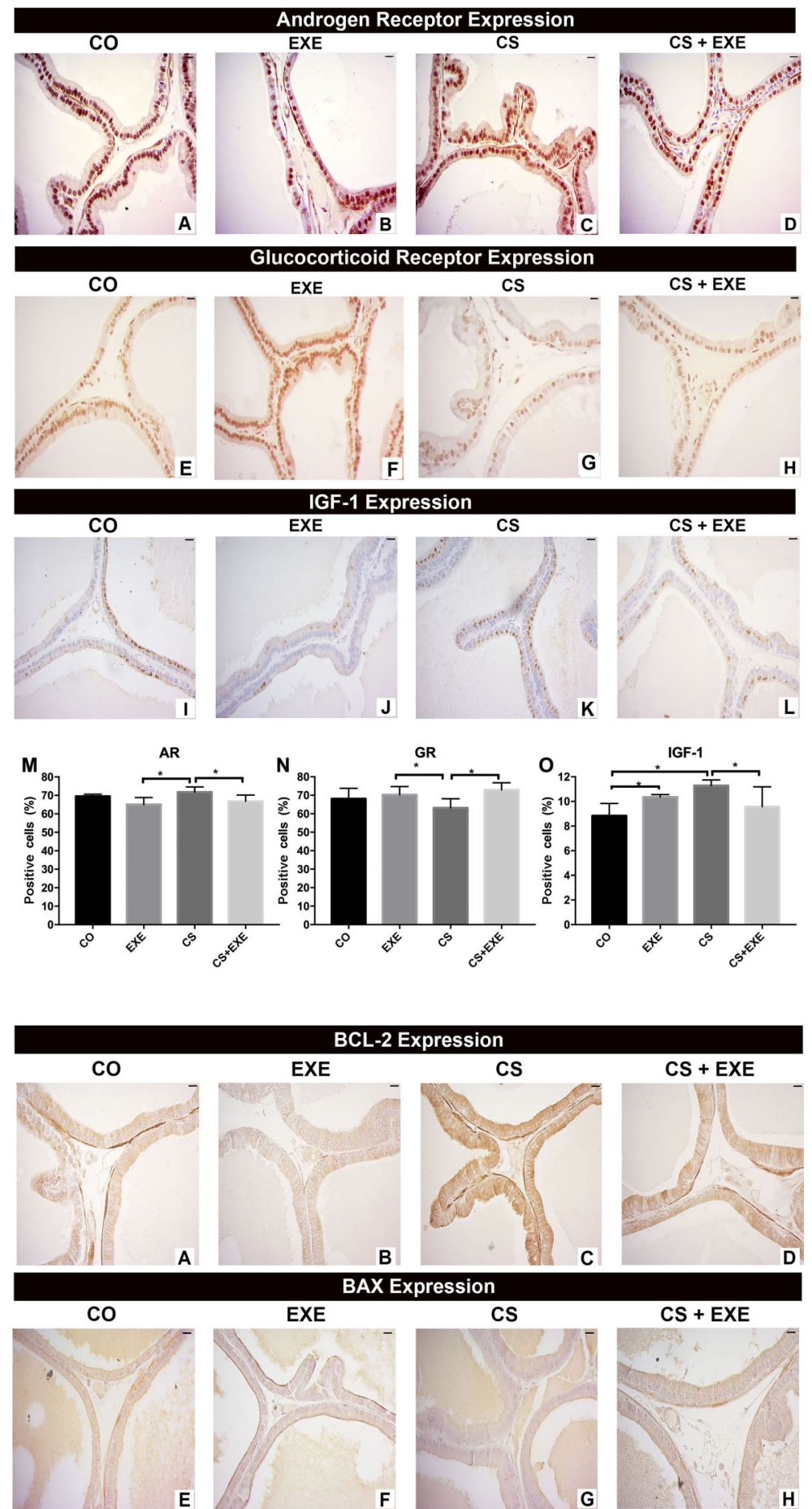

CS

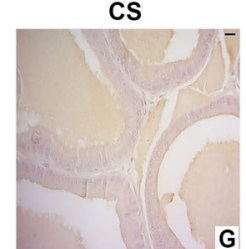

CS + EXE
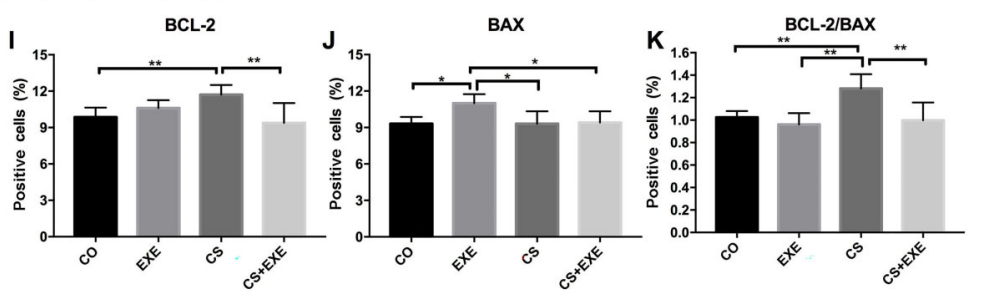

Figure 3. Distribution of the androgen receptor (AR), glucocorticoid receptors (GR), and insulinlike growth factor (IGF-1) expression evaluated by immunohistochemistry in the ventral prostate of Wistar rats submitted to cigarette smoke inhalation and aerobic exercise [control $(\mathrm{CO}) \mathrm{n}=10$; cigarette smoke inhalation (CS) $n=8$; exercise (EXE) $n=10$; cigarette smoke inhalation + exercise (CS + EXE) $\mathrm{n}=10]$. AR expression is represented in images $\mathbf{A}$ to $\mathbf{D}$ and quantification of AR-positive prostate cells is shown in graph $\mathbf{M}$. Representative images of GR expression are shown in images $\mathbf{E}$ to $\mathbf{H}$ and GR-positive cells are quantified in graph $\mathbf{N}$. Images I to $L$ are representative of IGF-1 expression, and the percentage of IGF-1-positive cells is shown in graph $\mathbf{O}$. Scale bars, $20 \mu \mathrm{m}$. Data are reported as mean percentage $\pm S D$. ${ }^{*} P<0.05$, one-way ANOVA with Tukey's multiple comparisons post hoc test.
Figure 4. Distribution of $B C L-2$ and $B A X$ protein expression evaluated through immunohistochemistry of the ventral prostate in Wistar rats undergoing inhalation of cigarette smoke and aerobic physical training [control $(\mathrm{CO}) \mathrm{n}=10$; cigarette smoke inhalation (CS) $n=8$; exercise (EXE) $n=10$; cigarette smoke inhalation + exercise (CS + EXE) $n=10]$. Representative images of BCL-2 and BAX expression are presented in $\mathbf{A}$ to $\mathbf{D}$ and $\mathbf{E}$ to $\mathbf{H}$, respectively. Scale bars, $20 \mu \mathrm{m}$. Graphs I and J show the quantification of positive prostate cells for BCL-2 and BAX, respectively. The ratio of BCL-2/ BAX expression in the prostate was quantified and is presented in $\mathbf{K}$. Data are reported as mean percentage of protein-positive tissue surface area \pm SD. ${ }^{*} \mathrm{P}<0.05$; ${ }^{* \star} \mathrm{P}<0.01$, one-way ANOVA with Tukey's multiple comparisons post hoc test. 

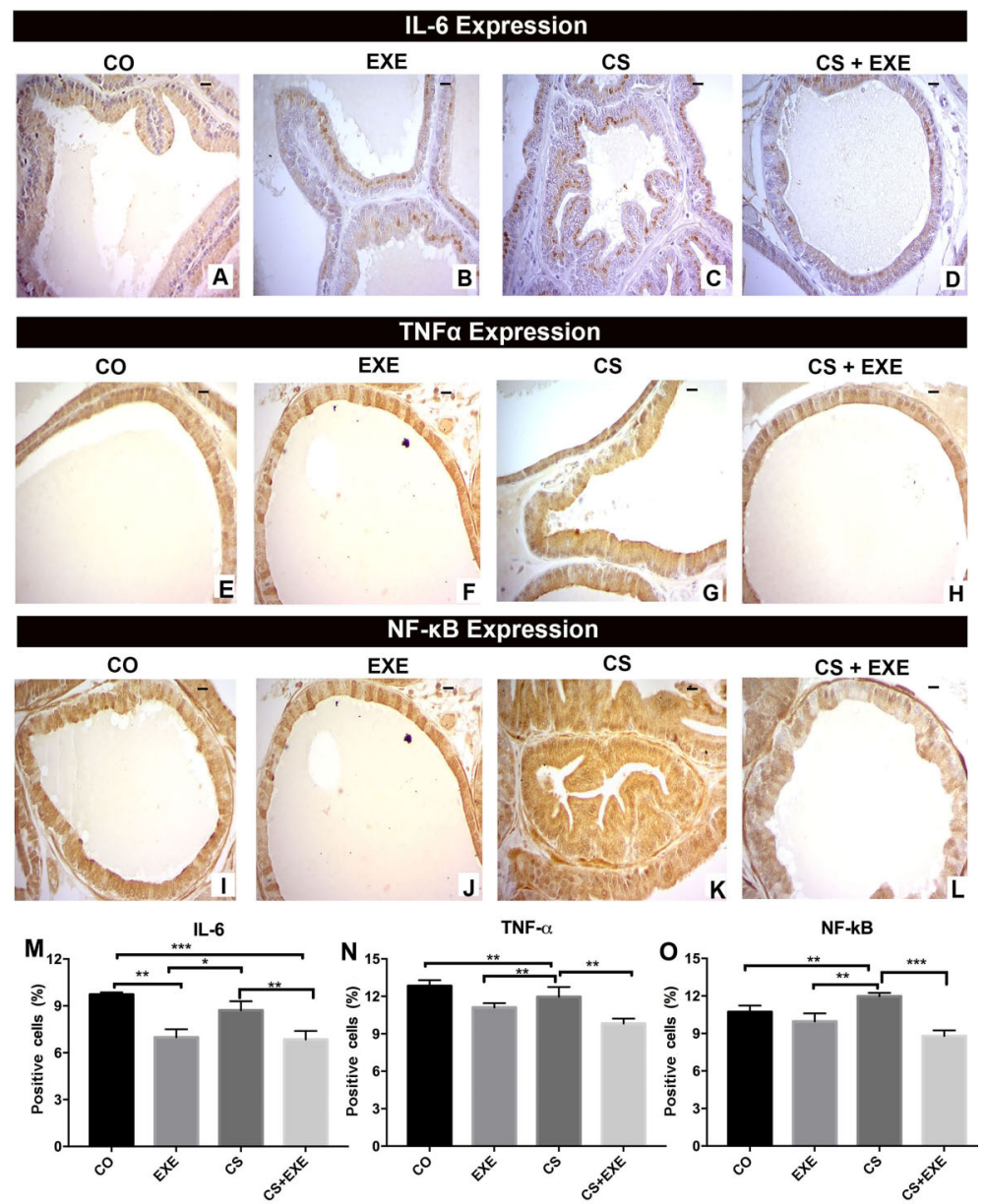

CS

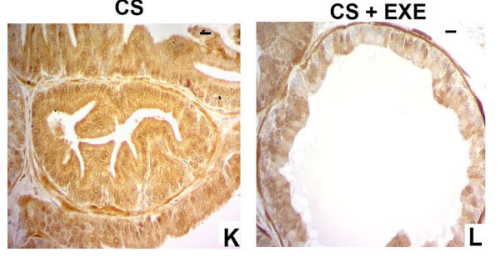

NF-kB

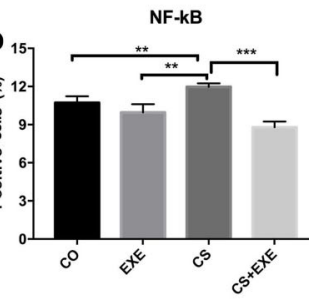

Figure 5. Distribution of interleukin (IL)-6, tumor necrosis factor-alpha (TNF- $\alpha$ ), and nuclear factorkappa $\mathrm{B}(\mathrm{NF}-\kappa \mathrm{B})$ protein in the ventral prostate of Wistar rats submitted to cigarette smoke inhalation and exercise as evaluated using immunohistochemistry [control $(\mathrm{CO}) \mathrm{n}=10$; cigarette smoke inhalation (CS) $n=8$; exercise (EXE) $n=10$; cigarette smoke inhalation + exercise $(C S+E X E) n=$ 10]. Representative images of IL-6, TNF- $\alpha$, and $N F-\kappa B$ are shown in images $\mathbf{A}$ to $\mathbf{D}, \mathbf{E}$ to $\mathbf{H}$, and $\mathbf{I}$ to $\mathbf{L}$, respectively. Scale bars, $20 \mu \mathrm{m}$. Graphs $\mathbf{M}, \mathbf{N}$, and $\mathbf{O}$ show the quantification of positive prostate cells for IL-6, TNF- $\alpha$, and NF- $\mathrm{B}$, respectively. Data are reported as mean percentages of proteinpositive tissue surface area $\pm S D$. ${ }^{*} P<0.05$; ${ }^{* *} \mathrm{P}<0.01,{ }^{* *} \mathrm{P}<0.001$, one-way ANOVA with Tukey's multiple comparisons post hoc test. physical training in both the CS + EXE and EXE groups reduced the expression of NF- $\kappa B$, TNF- $\alpha$, and IL- 6 , and increased GR expression.

\section{Discussion}

Our findings demonstrated that: a) cigarette smoke reduced food consumption, weight gain, and BMI values, and regulated food consumption and body weight; $b$ ) exercise increased blood triglyceride concentrations and decreased cholesterol and glucose, although smoke exposure increased cholesterol; c) smoke exposure upregulated AR and IGF-1 expression and reduced GR with concomitant increases in anti-apoptotic protein BCL-2 and prostate epithelium volume; d) pro-apoptotic protein BAX was upregulated following exercise and downregulated after smoke exposure; and e) exercise reduced inflammatory cytokines in the prostate, and smoke exposure increased the expression of NF- $\mathrm{BB}$. Thus, these results suggested that cigarette smoke inhalation promoted the expression of proliferative, anti-apoptotic, and inflammatory compounds in the prostate. These changes may be reversed with exercise, as aerobic physical training increased the expression of pro-apoptotic proteins and reduced inflammation, maintaining homeostasis in prostatic tissue. The proposed relationships between physical exercise, cigarette smoke exposure, prostatic health, and apoptotic and proliferative balance are summarized in Figure 6.

We observed changes suggesting that cigarette smoke exposure reduced weight gain by modulating feed efficiency, and consequently weight gain. The regulation of appetite and ingested energy are fundamental for maintaining caloric balance and body weight, and previous studies have also shown that administering cigarette smoke to rats reduces body weight (15). The anorexic effects of tobacco are attributed to nicotine (11). In contrast, aerobic physical exercise reduces BMI and improves feed efficiency in rats. However, cigarette smoke inhalation in combination with physical exercise reduces weight gain and feed efficiency, compared to exercise or smoke inhalation alone, even following high caloric intake. Both cigarette smoke and aerobic training increase metabolic rates and thermogenesis in adipose tissue, stimulate 

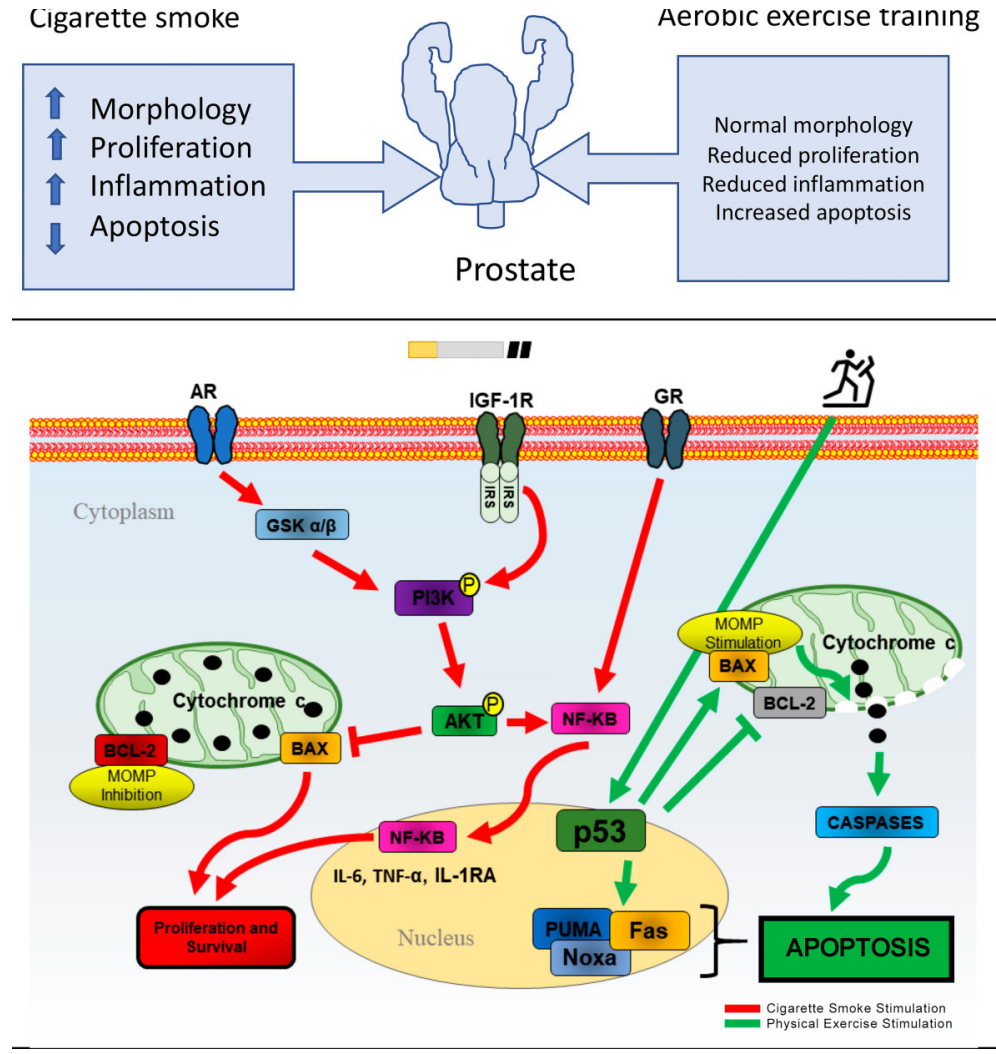

Figure 6. Central role of cigarette smoking on prostate inflammation in the progression of dysregulation and the effects of physical exercise. The prostate is a steroid- and immune-competent organ characterized by complex molecular pathways considered to be triggers for the dysregulation of the prostatic immune system and the development of chronic prostatic inflammation. Cigarette smoking promotes hormonal receptor and growth factor activation, which can trigger the phosphorylation of the PI3K and AKT pathways, inhibiting BAX and stimulating nuclear factorkappa B (NF-kB) activity. AKT prevents cytochrome $C$ release and inhibits apoptosis following cigarette smoking. Regular physical exercise can regulate the activation of the $\mathrm{PI} 3 \mathrm{~K} / \mathrm{AKF} / \mathrm{NF}-\mathrm{KB}$ pathway and promote glucocorticoid receptors (GR) activation by reducing NF- $\mathrm{KB}$ and cytokine expression, possibly by p53 activation and subsequent BAX upregulation, which leads to apoptosis and proliferative reduction. AR: androgen receptor; MOMP: mitochondrial outer membrane permeabilization. lipolysis, and reduce food intake, and may act on the recycling of fatty acids in triglycerides, thus contributing to lower body fat indexes (16). Nevertheless, the literature links cigarette smoking with visceral fat increase, enhanced cholesterol levels, greater waist-to-hip ratio, and atherosclerotic cardiovascular disease, and suggests that reduction in body weight by smoking is non-beneficial to health (17).

Smoking may promote dyslipidemia (18) as observed in this study showing that smoke inhalation increases cholesterol and glucose levels. In support of this, many studies have shown an association between dyslipidemia and benign prostatic hyperplasia (19). Mounting evidence suggests that metabolic syndrome is correlated with smoking (16). The relationship between smoking, dyslipidemia, and associated metabolic syndrome could play a role in the development and progression of prostate cancer (20). Mostaghel et al. (21) found that each $10 \mathrm{mg} /$ $\mathrm{dL}$ increase in total cholesterol above the abnormal cut-off value of $200 \mathrm{mg} / \mathrm{dL}$ was associated with a $9 \%$ increased risk of prostate cancer. Our results demonstrated that cigarette smoke exposure increased cholesterol, whereas aerobic physical training reduced cholesterol. MoralesPalomo and colleagues (22) found that aerobic exercise reduces metabolic syndrome by improving cardiorespiratory function. Thus, the effectiveness of physical training on reducing cholesterol levels reported in this study corroborated current literature findings (23). Aerobic exercise possibly recovered smoke-induced consequences on prostate health by reducing the effects associated with metabolic syndrome. The groups exposed to cigarette smoke showed a positive correlation between total cholesterol levels with prostatic epithelium $(r=0.554$, $\mathrm{P}=0.01)$ and $B C L-2$ expression ( $r=0.447, P=0.048)$. In summary, we found strong evidence to support the link between total cholesterol levels in serum and the risk of cell proliferation in the prostate.

Interestingly, aerobic physical training downregulated the expression of AR in prostate cells, which was associated with reduced proliferation in the prostatic epithelium. Cigarette smoke inhalation, in contrast, was associated with significantly higher AR, IGF-1, and BCL-2 expression in the prostate. High prostatic AR expression induced by cigarette smoke inhalation is associated with elevated levels of testosterone and decreased estradiol (24). Stone and colleagues (19) previously found that smokers with prostate cancer show higher than normal levels of plasmatic testosterone, which is associated with CYP3A expression, a proliferative inducer. Likewise, our findings showed that cigarette smoke exposure promoted proliferation in the prostate, potentially stimulated by $A R$ and IGF-1 activity. Increased testosterone-induced $A R$ expression may increase expression of the IGF-1 receptor (25); elevated IGF-1 expression activates the phosphorylation of subsequent proteins in the PI3K/AKT 
pathway (26)(32). Phosphorylation of AKT by AR and IGF-1 activity stimulates BCL-2 and BCL-XL, inhibiting the release of cytochrome $C$ from mitochondria and preventing cell apoptosis while promoting cell proliferation (26). These findings are further substantiated by our observations that prostatic proliferation was greater in animals following cigarette smoke inhalation than those that did not inhale smoke. Furthermore, physical exercise reduced activation of AR and IGF-1 in the prostate, possibly by reducing the expression of free testosterone (27).

Cigarette smoke, which induces oxidative stress, protease release, and drives inflammatory response, is considered a factor in the development of prostatic diseases (28). Previous findings show that GR expression is significantly lower in animals exposed to cigarette smoke, leading to increased transcription of pro-inflammatory genes (7). Considering that GR plays an integral role in the regulation of inflammatory responses in the prostate, we further investigated GR expression in response to cigarette smoke exposure and found reduced GR expression. GR may act via protein-protein interactions with other transcription factors such as activator protein-1, Smad3, and NF- $\mathrm{KB}(29)$ to indirectly alter gene transcription. Our previous work demonstrates that the antiinflammatory effects of GR activity occur through a DNA-independent mechanism (5). Glucocorticoids have been shown to block NF-kB activation by multiple mechanisms in several cell types (7), including binding of GR

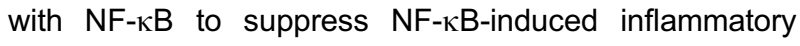
gene expression in the prostate. Our present results demonstrated that smoke inhalation reduced GR expression and increased NF- $\kappa \mathrm{B}$, despite no differences in IL-6 and TNF- $\alpha$ expression. The suppression of GR activity by p65 requires phosphorylation at $S 276$ by protein kinase $A$ and GR-mediated inhibition of NF- $\kappa B$ activity is also protein kinase A-dependent. Thus, the cross-suppression of NF- $\kappa B$ and GR activity is regulated by protein kinase A-associated signaling (30). However, physical exercise restores GR protein expression, which might subsequently attenuate smoking-induced inflammatory response and improve NF- $\mathrm{kB}$ and IL-6 activity in the prostate.

In the present study, we observed that chronic exposure to cigarette smoke-induced characteristics consistent with prostate cancer pathology and intraepithelial neoplasm, including increased epithelium volume, inflammation, and a proliferative state. Additionally, we found that aerobic physical exercise decreased the inflammatory proteins IL-6, TNF- $\alpha$, and NF- $\kappa B$ in prostate tissue. Petersen and Pedersen (31) suggested that during an inflammatory process, cytokines produced by adipose tissue such as TNF- $\alpha$ are recruited and stimulate the production of IL- 6 and the interleukin receptor antagonist-1. When produced and mediated during exercise, IL- 6 inhibits the effects of TNF- $\alpha$ and enhances expression of anti-inflammatory cytokines such as IL-10, inhibiting the production of inflammatory proteins such as IL- $1 \beta$, TNF- $\alpha$, and IL- $1 \alpha$.
Collectively, these results suggest that physical exercise is effective in protecting prostate function and suppressing the inflammatory response. Thus, we have demonstrated that physical exercise may promote apoptosis in prostatic cells by inhibiting androgen stimulation and the action of growth factors (5), further supported by alterations in BCL-2/BAX ratios seen here that suggested increased apoptosis. It has previously been established that BAX is a potent apoptosis inducer through the action of its target genes, such as p53. While we do not know the exact molecular mechanisms underlying apoptotic modulation in aerobic physical exercise, we hypothesized that exercise may stimulate p53 phosphorylation, increasing phosphorylated JNK and p38 MAPK, nuclear c-JUN, and activator protein-1 DNA-binding activity. In support of this, Camera et al. (32) found that physical exercise increases the expression of p38MAPK, p53, and PGC-1- $\alpha$. We observed that aerobic physical training directly or indirectly regulated apoptosis in the prostate as reflected by decreased BCL-2 levels compared to the cigarette smoke exposure-only group. These data suggest that physical exercise may protect against the development of tobaccoinduced prostate diseases.

There are some notable limitations to this study. Firstly, the animals in our study were exposed to an 8-week regimen of smoke exposure, and it is possible that a longer exposure period could produce more extensive changes to the prostate that would better recapitulate prostate morphology changes observed in clinical populations. Secondly, the molecular pathways in the prostate associated with aerobic physical exercise have yet to be identified, and this study did not explicitly evaluate the mechanisms associated with smoke- and exercise-related changes to prostate health. Additionally, as previously discussed, cigarette smoke exposure can induce oxidative stress in the prostate, which may impact the epithelium. However, our study did not evaluate the role of oxidative stress and p53 function associated with physical exercise. Future research should be conducted to increase understanding of how physical exercise and smoke exposure modulate prostate health at the molecular level, and how variables other than those investigated in this work could affect prostate health in the context of smoking and exercise.

In conclusion, our results demonstrated that cigarette smoke exposure altered biochemical parameters and exacerbated cell proliferation in the prostate. In contrast, aerobic exercise improved body and prostate weight, decreased AR and IGF-1, and regulated the BLC-2/BAX index towards increased apoptosis. These observations indicated that chronic exposure to cigarette smoke may anticipate the onset of prostatic diseases caused by increased cell proliferation, although regular exercise may maintain prostate tissue homeostasis. The expected outcomes of anti-tumor therapies are increased apoptosis and reduced cell proliferation. Physical exercise has 
demonstrated effective apoptotic action and, as seen in this study, anti-inflammatory effects in the prostate. Further research is needed to delineate the mechanisms mediated by aerobic physical exercise in the prostate and to determine optimal intensity, duration, and type of exercise to optimize the beneficial effects on cellular health and the prostate while minimizing the adverse effects of smoke exposure.

\section{References}

1. Sosnowski R, Bjurlin MA, Verze P, De Nunzio C, Shariat SF, Brausi $M$, et al. Role of cigarette smoking in urological malignancies and clinical interventions for smoking cessation. Cent Eur J Urol 2016; 69: 366-369, doi: 10.5173/ceju. 2016.883

2. Ypsilantis $P$, Politou M, Anagnostopoulos C, Kortsaris A, Simopoulos $C$. A rat model of cigarette smoke abuse liability. Comp Med 2012; 62: 395-399.

3. Egleton RD, Brown KC, Dasgupta P. Nicotinic acetylcholine receptors in cancer: multiple roles in proliferation and inhibition of apoptosis. Trends Pharmacol Sci 2008; 29: 151-158, doi: 10.1016/j.tips.2007.12.006.

4. Brookman-May SD, Campi R, Henríquez JDS, Klatte T, Langenhuijsen JF, Brausi M, et al. Latest evidence on the impact of smoking, sports, and sexual activity as modifiable lifestyle risk factors for prostate cancer incidence, recurrence, and progression: a systematic review of the literature by the European Association of Urology Section of Oncological Urology (ESOU). Eur Urol Focus 2019; 5: 756-787, doi: 10.1016/j.euf.2018.02.007.

5. Teixeira GR, Fávaro WJ, Pinheiro PF, Chuffa LG, Amorim JP, Mendes LO, et al. Physical exercise on the rat ventral prostate: Steroid hormone receptors, apoptosis and cell proliferation. Scand J Med Sci Sports 2012; 22: e86-e92, doi: 10.1111/j.1600-0838.2012.01501.x.

6. Dwivedi S, Goel A, Mandhani A, Khattri S, Pant KK. Tobacco exposure may enhance inflammation in prostate carcinoma patients: an explorative study in north Indian population. Toxicol Int 2012; 19: 310-318, doi: 10.4103/0971-6580.103681.

7. Scheschowitsch K, Leite JA, Assreuy J. New insights in glucocorticoid receptor signaling-more than just a ligandbinding receptor. Front Endocrinol (Lausanne) 2017; 8: 16, doi: 10.3389/fendo.2017.00016

8. Lee J, Taneja V, Vassallo R. Cigarette smoking and inflammation: cellular and molecular mechanisms. J Dent Res 2012; 91: 142-149, doi: 10.1177/0022034511421200.

9. Kenfield SA, Stampfer MJ, Chan JM, Giovannucci E. Smoking and prostate cancer survival and recurrence. JAMA 2011; 305: 2548-2555, doi: 10.1001/jama.2011.879.

10. Domingo-Domenech J, Mellado B, Ferrer B, Truan D, Codony-Servat J, Sauleda S, et al. Activation of nuclear factor-kB in human prostate carcinogenesis and association to biochemical relapse. Br J Cancer 2005; 93: 1285-1294, doi: 10.1038/sj.bjc.6602851.

11. Jessen A, Buemann B, Toubro S, Skovgaard IM, Astrup A. The appetite-suppressant effect of nicotine is enhanced by caffeine. Diabetes Obes Metab 2005; 7: 327-333, doi: 10.1111/j.1463-1326.2004.00389.x.

12. Ferreira JCB, Rolim NPL, Bartholomeu JB, Gobatto CA, Kokubun E, Brum PC. Maximal lactate steady state in

\section{Acknowledgments}

The authors thank the Coordenação de Aperfeiçoamento de Pessoal de Nível Superior-Brasil (CAPES), Finance Code - 001 and the São Paulo Research Foundation (FAPESP; Proc. 2013/08593-0) for financial support.

running mice: Effect of exercise training. Clin Exp Pharmacol Physiol 2007; 34: 760-765, doi: 10.1111/j.1440-1681.2007. 04635.x.

13. Novelli ELB, Diniz YS, Galhardi CM, Ebaid GMX, Rodrigues HG, Mani F, et al. Anthropometrical parameters and markers of obesity in rats. Lab Anim 2007; 41: 111-119, doi: 10.1258/ 002367707779399518.

14. Hewitt SM, Baskin DG, Frevert CW, Stahl WL, Rosa-Molinar E. Controls for Immunohistochemistry: The Histochemical Society's Standards of Practice for Validation of Immunohistochemical Assays. J Histochem Cytochem 2014; 62: 693-697, doi: 10.1369/0022155414545224.

15. Ypsilantis P, Politou M, Anagnostopoulos C, Tsigalou C, Kambouromiti G, Kortsaris A, et al. Effects of cigarette smoke exposure and its cessation on body weight, food intake and circulating leptin, and ghrelin levels in the rat. Nicotine Tob Res 2013; 15: 206-212, doi: 10.1093/ntr/ nts113.

16. Slagter SN, Vliet-Ostaptchouk JVV, Vonk JM, Boezen HM, Dullaart RPF, Kobold ACM, et al. Associations between smoking, components of metabolic syndrome and lipoprotein particle size. BMC Med 2013; 1: 195, doi: 10.1186/17417015-11-195.

17. Kim JH, Shim KW, Yoon YS, Lee SY, Kim SS, Oh SW. Cigarette Smoking Increases Abdominal and Visceral Obesity but Not Overall Fatness: An Observational Study. PLoS One 2012; 7: 5-9, doi: 10.1371/annotation/f6ebe3d3ef7c-42ce-86fe-d5a661d7f67f.

18. Craig WY, Palomaki GE, Haddow JE. Cigarette smoking and serum lipid and lipoprotein concentrations: an analysis of published data. BMJ 1989; 298: 784-788, doi: 10.1136/ bmj.298.6676.784.

19. Stone A, Ratnasinghe LD, Emerson GL, Modali R, Lehman T, Runnells G, et al. CYP3A43 Pro 340 Ala polymorphism and prostate cancer risk in African Americans and Caucasians. Cancer Epidemiol Biomarkers Prev 2005; 14: 1257-1261, doi: 10.1158/1055-9965.EPI-04-0534.

20. Rhee H, Vela I, Chung E. Metabolic Syndrome and Prostate Cancer: a Review of Complex Interplay Amongst Various Endocrine Factors in the Pathophysiology and Progression of Prostate Cancer. Horm Cancer 2016; 7: 75-83, doi: 10.1007/s12672-015-0238-x.

21. Mostaghel EA, Solomon KR, Pelton K, Freeman MR, Montgomery RB. Impact of circulating cholesterol levels on growth and intratumoral androgen concentration of prostate tumors. PLoS One 2012; 7: e30062, doi: 10.1371/journal. pone.0030062.

22. Morales-Palomo F, Ramirez-Jimenez M, Ortega JF, MoraRodriguez R. Effectiveness of aerobic exercise programs for health promotion in metabolic syndrome. Med Sci Sport 
Exerc 2019; 51: 1876-1883, doi: 10.1249/MSS.0000000000 001983.

23. Yu S, Xia S, Yang D, Wang K, Yeh S, Gao Z, et al. Androgen receptor in human prostate cancer-associated fibroblasts promotes prostate cancer epithelial cell growth and invasion. Med Oncol 2013; 30: 674, doi: 10.1007/s12032-013-0674-9.

24. Zeigler-Johnson CM, Walker AH, Mancke B, Spangler E, Jalloh $\mathrm{M}, \mathrm{McBride} S$, et al. Ethnic differences in the frequency of prostate cancer susceptibility alleles at SRD5A2 and CYP 3A4. Hum Hered 2002; 54:13-21, doi: 10.1159/000066695.

25. Heidegger I, Massoner P, Sampson N, Klocker H. The insulin-like growth factor (IGF) axis as an anticancer target in prostate cancer. Cancer Lett 2015; 367: 113-121, doi: 10. 1016/j.canlet.2015.07.026.

26. Asmarinah A, Paradowska-Dogan A, Kodariah R, Tanuhardja $B$, Waliszewski $P$, Mochtar $C A$, et al. Expression of the $\mathrm{Bcl}-2$ family genes and complexes involved in the mitochondrial transport in prostate cancer cells. Int $\mathrm{J}$ Oncol 2014; 45: 1489-1496, doi: 10.3892/ijo.2014.2576.

27. Kobayashi N, Barnard RJ, Said J, Hong-Gonzalez J, Corman $\mathrm{DM}, \mathrm{Ku} \mathrm{M}$, et al. Effect of low-fat diet on development of prostate cancer and Akt phosphorylation in the Hi-Myc Transgenic mouse model. Cancer Res 2008; 68: 30663073, doi: 10.1158/0008-5472.CAN-07-5616.

28. Tang B, Han CT, Gan HL, Zhang GM, Zhang CZ, Yang WY, et al. Smoking increased the risk of prostate cancer with grade group $\geqslant 4$ and intraductal carcinoma in a prospective biopsy cohort. Prostate 2017; 77:984-989, doi: 10.1002/ pros. 23354 .

29. Schäcke H, Döcke WD, Asadullah K. Mechanisms involved in the side effects of glucocorticoids. Pharmacol Ther 2002; 96: 23-43, doi: 10.1016/S0163-7258(02)00297-8.

30. Christian F, Smith EL, Carmody R.J. The regulation of NF$\kappa B$ subunits by phosphorylation. Cells 2016; 5: 12, doi: 10.3390/cells5010012.

31. Petersen AMW, Pedersen BK. The anti-inflammatory effect of exercise. J Appl Physiol 2005; 98: 1154-1162, doi: 10.1152/ japplphysiol.00164.2004.

32. Camera DM, Hawley JA, Coffey VG. Resistance exercise with low glycogen increases p53 phosphorylation and PGC$1 \alpha$ mRNA in skeletal muscle. Eur J Appl Physiol 2015; 115: 1185-1194, doi: 10.1007/s00421-015-3116-x. 